\title{
A NON-HYPOELLIPTIC DIRICHLET PROBLEM FROM STOCHASTICS
}

\author{
KARL DOPPEL and NIELS JACOB \\ To the memory of Rolf Nevanlinna
}

\section{Introduction}

1.1. In a recent paper [2] E. B. Dynkin solved a Dirichlet problem for a nonhypoelliptic differential operator $L(D)$ with methods of probability theory by extending some results of the theory of Markov processes.

This operator $L(D)$ is defined in the following way: Let $k$ be a positive integer. For each $j(1 \leqq j \leqq k)$ take $m_{j} \in N$ and set $n=m_{1}+\ldots+m_{k}$. With the notation

$$
l_{1}=0, \quad l_{j}=\sum_{i=1}^{j-1} m_{i} \quad(2 \leqq j \leqq k)
$$

we set

$$
\Delta_{j}:=\sum_{i=1}^{m_{j}} D_{l_{j}+i}^{2} \quad \text { with } \quad D_{l_{j}+i}=-\sqrt{-1} \frac{\partial}{\partial x_{l_{j}+i}} .
$$

Thus, $\Delta_{j}$ denotes the Laplace operator which acts on functions defined on subsets of $\boldsymbol{R}^{m_{j}} \subset \boldsymbol{R}^{n}$. We define the differential operator $L(D)$ by

$$
L(D):=\Delta_{1} \ldots \Delta_{k}
$$

The operator $L(D)$ is not hypoelliptic for $k>1$. This fact can be seen already in the simplest case where $k=2$ and $m_{1}=m_{2}=1$. Then

$$
L(D)=D_{1}^{2} D_{2}^{2}=\frac{\partial^{4}}{\partial x_{1}^{2} \partial x_{2}^{2}}
$$

and the polynomial associated with $L(D)$ is given by $P(\xi)=\xi_{1}^{2} \xi_{2}^{2}$. Since for $\xi_{1}=0$ and $\xi_{2}=n(n \in N)$ we have

$$
\frac{\partial^{2}}{\partial \xi_{1}^{2}} P(\xi)=2 n^{2},
$$


it follows by a result of L. Hörmander (see [6], p. 99) that $L(D)$ is not hypoelliptic. The usual Sobolev space methods are not applicable to a study of Dirichlet problems for the operator $L(D)$.

1.2. In this paper we present a method which can be understood as a generalization of the Hilbert space methods used in the elliptic theory. Here we use "anisotropic" Sobolev spaces which correspond in a natural way to the operator $L(D)$. It turns out that in these anisotropic spaces the behaviour of the non-hypoelliptic operator $L(D)$ is quite similar to that of an elliptic operator in the usual Sobolev spaces.

For a domain $G$ which is the Cartesian product of bounded open sets $G_{j} \subset \boldsymbol{R}^{m_{j}}$ $\left(n=m_{1}+\ldots+m_{k}\right)$ with sufficiently smooth boundaries $\partial G_{j}$ we show that a generalized Dirichlet problem, for which the solution is searched in such an anisotropic space, has a unique solution. Further one gets for a class of data a regularity result for this solution.

We will mention that Rolf Nevanlinna expressed a similar idea in unpublished lectures given in Ann Arbor, Helsinki and Zürich. He indicated the possibility to construct for a differential operator a suitable bilinear form such that solving Dirichlet problems for this operator can be reduced to the problem of orthogonal projection in the sense of this bilinear form (cf. [8] and also [1]).

1.3. We give a slightly modified representation of $L(D)$. We denote by $N_{0}^{n}$ the set of all ordered systems of $n$ nonnegative integers (multi-indices). For $\alpha=\left(\alpha_{1}, \ldots, \alpha_{n}\right) \in N_{0}^{n}$ we define its length as usual by $|\alpha|=\alpha_{1}+\ldots+\alpha_{n i}$. For $\sigma, \tau \in N_{0}^{n}$, $\sigma=\left(\sigma_{1}, \ldots, \sigma_{n}\right), \tau=\left(\tau_{1}, \ldots, \tau_{n}\right)$, we set $\sigma \leqq \tau$ if $\sigma_{i} \leqq \tau_{i}$ for $1 \leqq i \leqq n$. It is clear that with this definition $N_{0}^{n}$ is a partially ordered set.

Let $m_{j}$ and $l_{j}(1 \leqq j \leqq k)$ be defined as in 1.1. For each $j(1 \leqq j \leqq k)$ we define $m_{j}$ multi-indices $\varepsilon_{t_{j}} \in N_{0}^{n}$ of length $1,\left|\varepsilon_{t_{j}}\right|=1$, having its only nonvanishing coordinate in the $t_{j}$-th position, $l_{j}+1 \leqq t_{j} \leqq l_{j}+m_{j}$. Furthermore we set

and

$$
\Gamma=\left\{\alpha \mid \alpha \in N_{0}^{n}, \alpha=\sum_{j=1}^{k} \varepsilon_{t_{j}}\right\}
$$

$$
2 \Gamma=\left\{\beta \mid \beta \in N_{0}^{n}, \beta=2 \alpha, \alpha \in \Gamma\right\} .
$$

Note that both $\Gamma$ and $2 \Gamma$ have $m_{1} \ldots m_{k}$ elements. Now we obtain the very useful expression for the differential operator $L(D)$ :

where we used the abbreviation

$$
L(D)=\sum_{\alpha \in T} D^{2 \alpha}
$$

$$
D^{\alpha}=D_{1}^{\alpha} \ldots D_{n^{n}}^{\alpha}
$$

The authors want to thank E. B. Dynkin for leading their interest to these problems and I. S. Louhivaara for his suggestions while writing this paper. 


\section{The Hilbert spaces $H^{\Gamma}(G), H_{0}^{\Gamma}(G)$ and $H^{(k)}(G)$}

2.1. Let $G$ be a bounded open set in $\boldsymbol{R}^{n}$. As usual, let $C^{k}(G), k \in N_{0}$, be the linear space of all complex valued functions $u$ which are $k$ times continuously differentiable in $G$. By $C_{0}^{k}(G)$ we denote the space of all functions $u \in C^{k}(G)$ each having a compact support in $G$. We write also $C_{0}^{\infty}(G)=\bigcap_{k \in N_{0}} C_{0}^{k}(G)$. Further we define for $k \in N_{0}$

$$
C_{*}^{k}(G):=\left\{u\left|u \in C^{k}(G), D^{\alpha} u \in L^{2}(G), \alpha \in N_{0}^{n},\right| \alpha \mid \leqq k\right\} .
$$

In $C_{*}^{k}(G)$ we associate with the operator $L(D)$ the sesquilinear form

$$
B(u, v):=\sum_{\alpha \in \Gamma} \int_{G} \overline{D^{\alpha} u(x)} D^{\alpha} v(x) d x .
$$

We define

$$
(u, v)_{\Gamma}:=B(u, v)+(u, v)_{0},
$$

where $(\cdot, \cdot)_{0}$ denotes the scalar product in $L^{2}(G)$,

$$
(u, v)_{0}=\int_{G} \overline{u(x)} v(x) d x .
$$

Thus, we have a scalar product $(\cdot, \cdot)_{\Gamma}$ on $C_{*}^{k}(G)$ with the corresponding norm $\|\cdot\|_{\Gamma}$. The completion of $C_{*}^{k}(G)$ with respect to the scalar product (2.2) will be denoted by $H^{\Gamma}(G)$. One obtains $H^{2 \Gamma}(G)$ analogously. For the closure of $C_{0}^{\infty}(G)$ in $H^{\Gamma}(G)$ we write $H_{0}^{\Gamma}(G)$. The elements of $H_{0}^{\Gamma}(G)$ are interpreted as functions with generalized homogenous boundary data (cf. Theorem 4).

In contrast to the usual Sobolev space theory we cannot conclude that the strong $L^{2}$-derivative $D^{\tau} u$ exists for $u \in H^{\Gamma}(G)$ and for each multi-index $\tau \in N_{0}^{n}$ with $\tau \leqq \sigma$ for some $\sigma \in \Gamma$. But one can prove (cf. also [7])

Lemma 1. Let $u \in H_{0}^{T}(G)$ be given. Then the strong $L^{2}$-derivative $D^{\tau} u$ exists for all multi-indices $\tau$ with $\tau \leqq \sigma$ for some $\sigma \in \Gamma$.

Proof. For $\tau \in \Gamma$ the assertion is obvious. Let now $\tau \notin \Gamma$ such that $\tau \leqq \sigma \in \Gamma$. Since $G \subset \boldsymbol{R}^{n}$ is a bounded set, we can find a constant $d>0$ such that for each $x=\left(x_{1}, \ldots, x_{n}\right) \in G$

$$
\max _{1 \leqq j \leqq n}\left|x_{j}\right| \leqq d
$$

holds. For $\varphi \in C_{0}^{\infty}(G)$ we get by partial integration $(1 \leqq j \leqq k)$

$$
\begin{gathered}
\left\|D^{\tau} \varphi\right\|_{0}^{2}=\int_{G}\left|D^{\tau} \varphi(x)\right|^{2} d x=-\int_{G} x_{j} \frac{\partial}{\partial x_{j}}\left(\overline{D^{\tau} \varphi(x)} D^{\tau} \varphi(x)\right) d x \\
\leqq 2 d\left\|D^{\tau+\varepsilon_{j}} \varphi\right\|_{0}\left\|D^{\tau} \varphi\right\|_{0} .
\end{gathered}
$$

Hence one has

$$
\left\|D^{\tau} \varphi\right\|_{0} \leqq 2 d\left\|D^{\tau+\varepsilon_{j}} \varphi\right\|_{0} .
$$


Since $\tau \leqq \sigma$, there exists a multi-index $\beta$ with $\tau+\sigma=\beta$ and the result follows by iteration.

As an immediate consequence of the Poincaré inequality we have

Lemma 2. On $H_{0}^{T}(G)$ the sesquilinear form (2.1) defines a scalar product equivalent to (2.2).

Proof. It is sufficient to prove all estimates for elements of $C_{0}^{\infty}(G)$. By (2.2) we have for all $\varphi \in C_{0}^{\infty}(G)$

$$
B(\varphi, \varphi) \leqq(\varphi, \varphi)_{\Gamma} .
$$

Now by the Poincaré inequality (cf. [5], p. 33) we get for each multi-index $\varepsilon_{j} \in N_{0}^{n}$, $\left|\varepsilon_{j}\right|=1(1 \leqq j \leqq n)$

$$
\|\varphi\|_{0}^{2} \leqq c_{1}\left\|D^{\varepsilon_{j}} \varphi\right\|_{0}^{2}
$$

where the constant $c_{1}$ depends only on $G$. Repeating this argument we get finally for each $\alpha \in \Gamma$

and by $(2.1)$

$$
\|\varphi\|_{0}^{2} \leqq c_{\alpha}\left\|D^{\alpha} \varphi\right\|_{0}^{2}
$$

which proves Lemma 2.

$$
\|\varphi\|_{0}^{2} \leqq c B(\varphi, \varphi),
$$

Thus we have on $H_{0}^{\Gamma}(G)$ a norm $\|\mid \cdot\|_{\Gamma}$ defined by

$$
\|\mid \varphi\|_{\Gamma}:=(B(\varphi, \varphi))^{1 / 2}, \quad \varphi \in C_{0}^{\infty}(G),
$$

equivalent to $\|\cdot\|_{\Gamma}$. This norm can be interpreted as the energetic norm associated with the differential operator $L(D)$.

2.2. Now let us suppose that the set $G \subset \boldsymbol{R}^{n}$ has

Property P. The open bounded set $G$ is the Cartesian product

$$
G=G_{1} \times \ldots \times G_{k}
$$

of bounded open sets $G_{j} \subset \boldsymbol{R}^{m_{j}}(1 \leqq j \leqq k), m_{1}+\ldots+m_{k}=n$, with sufficiently smooth boundaries $^{1} \partial G_{j}$.

We will generalize a well-known theorem from the theory of Sobolev spaces.

Let $G_{j}^{\prime} \subset \boldsymbol{R}^{m_{j}}(1 \leqq j \leqq k)$ be a bounded open set. If $\Phi_{j}$ is a diffeomorphism of class $C^{1}$ from $\bar{G}_{j}^{\prime}$ onto $\bar{G}_{j}$ then the tensor product $\Phi$,

$$
\Phi:=\Phi_{1} \otimes \ldots \otimes \Phi_{k},
$$

is a diffeomorphism of class $C^{1}$ from $\bar{G}^{\prime}=\bar{G}_{1}^{\prime} \times \ldots \times \bar{G}_{k}^{\prime}$ onto $\bar{G}=\bar{G}_{1} \times \ldots \times \bar{G}_{k}$.

\footnotetext{
${ }^{1}$ All our considerations are valid if the boundaries $\partial G_{j}$ are of class $C^{\infty}$ (cf. e.g. [3], pp. 9-10).
} 
By definition the Jacobi matrix is the direct sum of the linear mappings $A_{j}: \boldsymbol{R}^{m_{j}} \rightarrow \boldsymbol{R}^{m_{j}}$ $(1 \leqq j \leqq k)$ defined by

$$
A_{j}=\left(\begin{array}{ccc}
\frac{\partial x_{l_{j}+1}}{\partial y_{l_{j}+1}} & \cdots & \frac{\partial x_{l_{j}+m_{j}}}{\partial y_{l_{j}+1}} \\
\vdots & & \vdots \\
\frac{\partial x_{l_{j}+1}}{\partial y_{l_{j}+m_{j}}} & \cdots & \frac{\partial x_{l_{j}+m_{j}}}{\partial y_{l_{j}+m_{j}}}
\end{array}\right),
$$

where $x_{i}$ denotes the $i$-th component of $\Phi$. We remark that the elements of $A_{j}$ are continuous functions on $\bar{G}_{j}^{\prime}$.

Lemma 3. Let $G$ be a bounded open set with Property $\mathrm{P}$ and let $\Phi$ be defined by (2.6). Then for all $u \in H_{0}^{\Gamma}(G)$ the function

$$
u^{\prime}(y)=u(\Phi(y))
$$

is in $H_{0}^{\Gamma}\left(G^{\prime}\right)$ and

$$
c_{1}\left\|u^{\prime}\right\|_{\Gamma, G^{\prime}} \leqq\|u\|_{\Gamma, G} \leqq c_{2}\left\|u^{\prime}\right\|_{\Gamma, G^{\prime}}
$$

holds, where the constants $c_{1}, c_{2}$ depend only on the diffeomorphism $\Phi$.

Proof. By Lemma 2 inequality (2.9) is equivalent to

$$
\tilde{c}_{1}\left|\left\|u^{\prime}\left|\left\|_{\Gamma, G^{\prime}} \leqq\right\| u\|\|_{\Gamma, G} \leqq \tilde{c}_{2}\right|\right\| u^{\prime}\right| \|_{\Gamma, G^{\prime}}
$$

Now by definition we get for each function (2.8)

$$
\left\|\left.\left|u^{\prime} \|_{\Gamma, G^{\prime}}^{2}=\sum_{\alpha \in \Gamma} \int_{G^{\prime}}\right| D_{y}^{\alpha} u^{\prime}(y)\right|^{2} d y .\right.
$$

For a fixed $\alpha \in \Gamma$ we have by (1.3)

$$
\alpha=\varepsilon_{t_{1}}+\ldots+\varepsilon_{t_{k}},
$$

where $\varepsilon_{t_{j}}(1 \leqq j \leqq k)$ has its only non-vanishing coordinate in the $t_{j}$-th position, $l_{j}+1 \leqq t_{j} \leqq l_{j}+m_{j}$. Furthermore we get

$$
\begin{gathered}
D_{y}^{\alpha} u^{\prime}(y)=(-\sqrt{-1})^{k} \frac{\partial^{k}}{\partial y_{t_{1}} \ldots \partial y_{t_{k}}}(y) \\
=(-\sqrt{-1})^{k} \sum_{i_{1}=1}^{m_{1}} \ldots \sum_{i_{k}=1}^{m_{k}} \frac{\partial^{k} u(\Phi(y))}{\partial x_{l_{1}+i_{1}} \ldots \partial x_{l_{k}+i_{k}}} \frac{\partial x_{l_{1}+i_{1}}}{\partial y_{t_{1}}} \ldots \frac{\partial x_{l_{k}+i_{k}}}{\partial y_{t_{k}}} .
\end{gathered}
$$

Thus, using the fact that the Jacobian of $\Phi$ is continuous on $\bar{G}^{\prime}$, we get

$$
\left|D_{y}^{\alpha} u^{\prime}(y)\right| \leqq \hat{c}_{\alpha} \sum_{i_{1}=1}^{m_{1}} \ldots \sum_{i_{k}=1}^{m_{k}}\left|\frac{\partial^{k} u(\Phi(y))}{\partial x_{l_{1}+i_{1}} \ldots \partial x_{l_{k}+i_{k}}}\right|
$$


where the constant $\hat{c}_{\alpha}$ depends only on $\Phi$ and $\alpha$. Now one has with another constant $\tilde{c}_{\alpha}$

and further

$$
\begin{aligned}
\left|D_{y}^{\alpha} u^{\prime}(y)\right|^{2} & \leqq \hat{c}_{\alpha}^{2}\left(\sum_{i_{1}=1}^{m_{1}} \ldots \sum_{i_{k}=1}^{m_{k}}\left|\frac{\partial^{k} u(\Phi(y))}{\partial x_{l_{1}+i_{1}} \ldots \partial x_{l_{k}+i_{k}}}\right|\right)^{2} \\
& \leqq \tilde{c}_{\alpha}^{2} \sum_{i_{1}=1}^{m_{1}} \ldots \sum_{i_{k}=1}^{m_{k}}\left|\frac{\partial^{k} u(\Phi(y))}{\partial x_{l_{1}+i_{1}} \ldots \partial x_{l_{k}+i_{k}}}\right|^{2}
\end{aligned}
$$

$$
\left\|D_{y}^{\alpha} u^{\prime}\right\|_{0, G^{\prime}}^{2} \leqq \tilde{c}_{\alpha}^{2} \sum_{i_{1}=1}^{m_{1}} \ldots \sum_{i_{k}=1}^{m_{k}} \int_{G^{\prime}}\left|\frac{\partial^{k} u(\Phi(y))}{\partial x_{l_{1}+i_{1}} \ldots \partial x_{l_{k}+i_{k}}}\right|^{2} d y .
$$

We substitute $y=\Phi^{-1}(x)$. As the Jacobian of $\Phi^{-1}$ is continuous on $\bar{G}$ we find

$$
\left\|D_{y}^{\alpha} u^{\prime}\right\|_{0, G^{\prime}}^{2} \leqq c_{\alpha}^{2} \sum_{i_{1}=1}^{m_{1}} \ldots \sum_{i_{k}=1}^{m_{k}}\left\|\frac{\partial^{k} u}{\partial x_{l_{1}+i_{1}} \ldots \partial x_{l_{k}+i_{k}}}\right\|_{0, G}^{2}
$$

with a suitable constant $c_{\alpha}$. In the summation on the right side of (2.13) only multiindices which belong to $\Gamma$ appear, wherefore we get

$$
\left\|D_{y}^{\alpha} u^{\prime}\right\|_{0, G^{\prime}}^{2} \leqq c_{\alpha}^{2}\|\| u \|_{\Gamma, G}^{2} .
$$

Because (2.14) holds for all multi-indices $\alpha \in \Gamma$, we can finally show that there exists a constant $\tilde{c}_{1}^{-1}$ depending only on $\Phi$ such that

$$
\left|\left\|u^{\prime}||_{\Gamma, G^{\prime}} \leqq \tilde{c}_{1}^{-1}\left|\|u \mid\|_{\Gamma, G}\right.\right.\right.
$$

holds for all functions (2.8). By interchanging the roles of $x$ and $y$ we get the second inequality of (2.10).

To prove a theorem for functions in $H_{0}^{\Gamma}(G)$ which is quite similar to a theorem on the behaviour of the elements of $H_{0}^{l}(G)$ (cf. e.g. [5], p. 28), we define for each $j(1 \leqq j \leqq k)$

(for $\gamma \in \Gamma^{j}$ one has $|\gamma|=k-1$ ) and

$$
\Gamma^{j}:=\left\{\gamma \mid \gamma \in N_{0}^{n}, \gamma=\sum_{\substack{i=1 \\ i \neq j}}^{k} \varepsilon_{t_{i}}\right\}
$$

$$
\partial_{j} G:=G_{1} \times \ldots \times G_{j-1} \times \partial G_{j} \times G_{j+1} \times \ldots \times G_{k} .
$$

Theorem 4. Let $G$ be a bounded open set with Property P. Then for each $u \in H_{0}^{\Gamma}(G) \cap C^{k-1}(\bar{G})$ the relation

$$
\left.D^{\beta} u\right|_{\partial_{j} G}=0
$$

holds for all multi-indices $\beta$ with $\beta \leqq \gamma$ for some $\gamma \in \Gamma^{j}(1 \leqq j \leqq k)$.

Proof. It is obviously enough to prove the result for $j=1$. For a point $(\xi, \eta) \in \partial G_{1} \times G^{\sim}, G^{\sim}=G_{2} \times \ldots \times G_{k}$, we can assume by Lemma 3 that the point $\xi\left(\epsilon \partial G_{1}\right)$ and for a neighbourhood $U \subset \boldsymbol{R}^{m_{1}}$ of $\xi$ the set $U \cap \partial G_{1}$ lie in the hyper- 
surface $x_{1}=0$ and that $U \cap G_{1}$ lies in the halfspace $x_{1} \leqq 0^{2}$. In the Cartesian product $\left(U \cap \partial G_{1}\right) \times G^{\sim}$ we can find an $n$-dimensional cylinder $S_{h}$ with height $h$, the base of which is an $(n-1)$-dimensional ball

$$
B_{r_{h}}(\xi, \eta)=\left\{x\left|x \in \boldsymbol{R}^{n}, x_{1}=0, \sum_{i=2}^{m_{1}}\right| x_{i}-\left.\xi_{i}\right|^{2}+\sum_{i=m_{1}+1}^{n}\left|x_{i}-\eta_{i}\right|^{2}<r_{h}^{2}\right\}
$$

in $U \cap \partial G_{1}$ in the hypersurface $x_{1}=0$, where the radius $r_{h}$ is chosen such that the volume of $S_{h}$ is equal to $h^{2}$. With sufficiently small values of $h$ we have $S_{h} \subset$ $G_{1} \times G^{\sim}$.

For each $\varphi \in C_{0}^{\infty}(G)$ one has

$$
D^{\beta} \varphi(x)=0
$$

for all $x \in B_{r_{h}}(\xi, \eta)$ and all $\beta \in N_{0}^{n}$. From (2.17) one has

$$
D_{x}^{\beta} \varphi(x)=\int_{0}^{x_{1}} \frac{\partial}{\partial t} D_{x}^{\beta} \varphi\left(t, x_{2}, \ldots, x_{n}\right) d t .
$$

By the Cauchy-Schwarz inequality we get

$$
\left|D_{x}^{\beta} \varphi(x)\right|^{2} \leqq h \int_{0}^{h}\left|\frac{\partial}{\partial t} D_{x}^{\beta} \varphi\left(t, x_{2}, \ldots, x_{n}\right)\right|^{2} d t .
$$

Integration with respect to $x$ gives

$$
\int_{S_{h}}\left|D_{x}^{\beta} \varphi(x)\right|^{2} d x \leqq h^{2} \int_{S_{h}}\left|\frac{\partial}{\partial x_{1}} D_{x}^{\beta} \varphi(x)\right|^{2} d x .
$$

Since vol $S_{h}=h^{2}$, we get

$$
\frac{1}{\operatorname{vol} S_{h}} \int_{S_{h}}\left|D_{x}^{\beta} \varphi(x)\right| d x \leqq \int_{S_{h}}\left|D_{x}^{\beta+\varepsilon_{1}} \varphi(x)\right|^{2} d x
$$

for $\varepsilon_{1}=(1,0, \ldots, 0) \in \boldsymbol{N}_{0}^{n}$.

Now take a multi-index $\beta$ with $\beta \leqq \gamma$ for some $\gamma \in \Gamma^{1}$. Then one has $\beta+\varepsilon_{1} \leqq \gamma+\varepsilon_{1} \in \Gamma$. By Lemma 1 it follows that the right-hand integral tends to zero also for all elements $u \in H_{0}^{\Gamma}(G)$ if $h \rightarrow 0$, whence we have

$$
\lim _{h \rightarrow 0} \frac{1}{\operatorname{vol} S_{h}} \int_{S_{h}}\left|D^{\beta} u(x)\right|^{2} d x=0 .
$$

If in addition $u \in C^{k-1}(\bar{G})$, we conclude by using the mean value theorem

$$
D^{\beta} u(\xi, \eta)=0
$$

for all $\eta \in G^{\sim}$ and for all $\beta \leqq \gamma \in \Gamma^{1}$. This proves Theorem 4 .

${ }^{2}$ To reach this situation by a local $C^{1}$-diffeomorphism (cf. Lemma 3 ) we must have some regularity of the boundaries; in the special case $m_{1}=\ldots=m_{k}=1$ the following proof is always applicable without any transformation by a $C^{1}$-diffeomorphism. 
2.3. In this connection we remark that partial integration is possible for func tions of the set

$$
X:=H_{0}^{\Gamma}(G) \cap C^{\infty}(\bar{G}),
$$

provided that the set $G$ has Property P.

Lemma 5. Let $G$ be a bounded open set with Property P. Then

$$
\int_{\mathbf{G}} \overline{D^{\alpha} u(x)} D^{\alpha} v(x) d x=\int_{G} \overline{D^{2 \alpha} u(x)} v(x) d x
$$

holds for all $u, v \in X$ and $\alpha \in \Gamma$.

2.4. In the case where the bounded open set $G$ has Property $\mathrm{P}$ we introduce another Hilbert space denoted by $H^{(k)}(G)$. On $X$ a scalar product is defined by

$$
(u, v)_{(k)}:=(L(D) u, L(D) v)_{0}+(u, v)_{0} .
$$

The Hilbert space $H^{(k)}(G)$ is defined as the completion of $X$ with respect to the scalar product (2.21). It is clear that (2.21) gives the graph norm $\|\cdot\|_{(k)}$,

$$
\|u\|_{(k)}^{2}=\|L(D) u\|_{0}^{2}+\|u\|_{0}^{2}
$$

on $X$.

We consider the densely defined linear operator $L$ in the Hilbert space $L^{2}(G)$ given by

$$
\left\{\begin{array}{l}
D(L):=X\left(\subset L^{2}(G)\right), \\
L u:=L(D) u \text { for all } u \in X .
\end{array}\right.
$$

By partial integration one gets

$$
(L u, v)_{0}=\int_{G} \overline{L(D) u(x)} v(x) d x=\int_{G} \overline{u(x)} L(D) v(x) d x=(u, g)_{0}
$$

for $u, v \in X$, with $L(D) v=: g=: L^{*} v$. Thus, we have $D\left(L^{*}\right) \supset X$, and the adjoint operator $L^{*}$ is densely defined on $L^{2}(G)$. Hence the operator $L$ is closable with the closure (smallest closed extension) $L^{\sim}=L^{* *}$.

Theorem 6. Let $G$ be a bounded open set with Property P. Then the relation

holds.

$$
D\left(L^{\sim}\right)=H^{(k)}(G)
$$

Proof. We denote the graphs of $L$ and $L^{\sim}$ by

and

$$
G(L):=\{(u, L u) \mid u \in X\}
$$

$$
G\left(L^{\sim}\right):=\left\{\left(f, L^{\sim} f\right) \mid f \in D\left(L^{\sim}\right)\right\},
$$


respectively. On the other hand, it is well-known (cf. [10], p. 89) that $G\left(L^{\sim}\right)$ coincides with the completion of $G(L)$ with respect to the graph norm (2.22),

$$
G\left(L^{\sim}\right)=\overline{G(L)}\|\cdot\|_{(k)} \text {. }
$$

For an arbitrary $u \in H^{(k)}(G)$ there exists by definition a sequence $\left\{u_{m}\right\}_{m \in N} \subset X$ such that

$$
\left\|u_{m}-u\right\|_{0} \rightarrow 0 \text { for } m \rightarrow \infty
$$

and $\left\{L u_{m}\right\}_{m \in N}$ is a Cauchy sequence in $L^{2}(G)$, which implies the existence of a unique element $w \in L^{2}(G)$ such that

$$
\left\|L u_{m}-w\right\|_{0} \rightarrow 0 \text { for } m \rightarrow \infty .
$$

Because of (2.25) it follows that $(u, w) \in G\left(L^{\sim}\right)$, i.e., $u \in D\left(L^{\sim}\right)$ (and $w=L^{\sim} u$ ).

On the other hand, let $u \in D\left(L^{\sim}\right)$ be given. By the definition of a closed operator there exists a sequence $\left\{u_{m}\right\}_{m \in N} \subset D(L)=X$ such that (2.26) and (2.27) hold with $w:=L^{\sim} u$. By definition we have $u \in H^{(k)}(G)$.

Remark. 1. From Theorem 6 we obtain for the scalar product of $H^{(k)}(G)$ the expression

$$
(u, v)_{(k)}=\left(L^{\sim} u, L^{\sim} v\right)_{0}+(u, v)_{0} .
$$

2. Let $B$ be the sesquilinear from (2.1). By partial integration one has

$$
B(u, \varphi)=(L u, \varphi)_{0} \text { for all } u \in X \text { and } \varphi \in C_{0}^{\infty}(G)
$$

and by continuous extension

$$
B(u, v)=(L u, v)_{0} \text { for all } u, v \in X .
$$

By the Cauchy-Schwarz inequality we get

and especially

$$
|B(u, v)| \leqq\|u\|_{(k)}\|v\|_{0} \quad \text { for all } u, v \in X
$$

$$
\|\mid u\|\left\|_{\Gamma}^{2} \leqq\right\| u\left\|_{(k)}\right\| u \|_{0} \text { for all } u \in X .
$$

2.5. Now we will show that the elements of $H^{(k)}(G)$ have the same boundary behaviour as the elements of $H_{0}^{\Gamma}(G)$ :

$$
H^{(k)}(G) \cap H_{0}^{\Gamma}(G)=H^{(k)}(G) .
$$

Take an element $v \in H^{(k)}(G)$. By the definition of $H^{(k)}(G)$ there exists a sequence $\left\{u_{j}\right\} \subset X=H_{0}^{\Gamma}(G) \cap C^{\infty}(\bar{G})$ with

$$
\left\|u_{j}-v\right\|_{(k)}^{2}=\left\|L u_{j}-L^{\sim} v\right\|_{0}^{2}+\left\|u_{j}-v\right\|_{0}^{2} \rightarrow 0 .
$$

Especially $\left\{u_{j}\right\}$ is a Cauchy sequence in $H^{(k)}(G)$ and by $(2.29)$ also in $H_{0}^{\Gamma}(G)$ :

$$
\left\|u_{j}-u_{l}\right\|_{\Gamma} \rightarrow 0
$$


Thus $\left\{u_{j}\right\}$ has a limit element $v^{*}$ in $H_{0}^{\Gamma}(G)$ :

$$
\| u_{j}-v^{*}||_{r} \rightarrow 0 \text {. }
$$

By Lemma 2 it follows $\left\|u_{j}-v^{*}\right\|_{0} \rightarrow 0$. Hence we get $v=v^{*} \in H_{0}^{I}(G)$, which means $H^{(k)}(G) \subset H_{0}^{\Gamma}(G)$. This proves the assertion (2.30).

\section{A generalized Dirichlet problem}

3.1. Let $G$ be a bounded open set with Property P. We have defined the sesquilinear form $B$ on $C_{*}^{k}(G)$ by (2.1). Since $C_{*}^{k}(G)$ is dense in $H^{\Gamma}(G)$ and since the obvious estimate

$$
|B(u, v)| \leqq c\|u\|_{\Gamma}\|v\|_{\Gamma}
$$

holds for all $u, v \in C_{*}^{k}(G)$, this sesquilinear form can be extended continuously onto $H^{T}(G)$.

Using this sesquilinear form we can now formulate (analogously to the theory of strongly elliptic boundary value problems) a generalized Dirichlet problem. Starting from the Dirichlet problem of classical type

$$
\begin{gathered}
L(D) u=f \text { in } G, \\
D^{\alpha} u=g_{\alpha, j} \text { on } \partial_{j} G,
\end{gathered}
$$

for all $\alpha \in N_{0}^{n}, \alpha \leqq \gamma$ with some $\gamma \in \Gamma^{j}(1 \leqq j \leqq k)$ we pose the generalized problem as follows (cf. also [4]):

Problem 1. For $f \in L^{2}(G)$ and $g \in H^{T}(G)$ find all $u \in H^{T}(G)$ such that

$$
B(u, \varphi)=(f, \varphi)_{0}
$$

holds for all $\varphi \in C_{0}^{\infty}(G)$ and the generalized boundary condition

is fulfilled.

$$
v:=u-g \in H_{0}^{\Gamma}(G)
$$

From Lemma 2 it follows that the linear functional $l_{f, g}$ defined by

$$
l_{f, g}(\varphi):=(f, \varphi)_{0}-B(g, \varphi) \text { for all } \varphi \in C_{0}^{\infty}(G)
$$

is bounded on $H_{0}^{\Gamma}(G)$. Thus, Problem 1 is equivalent to

Problem 2. For $f \in L^{2}(G)$ and $g \in H^{\Gamma}(G)$ find all elements $v \in H_{0}^{\Gamma}(G)$ such that

holds for all $\varphi \in C_{0}^{\infty}(G)$.

$$
l_{f, g}(\varphi)=B(v, \varphi)
$$

3.2. Now, we have

Theorem 7. Problem 2 has a unique solution $v \in H_{0}^{\Gamma}(G)$. 
Proof. By Lemma 2 the sesquilinear form $B$ is equivalent to the scalar product (2.2) on $H_{0}^{\Gamma}(G)$. By the Fréchet-Riesz representation theorem the result follows immediately.

Remark. If (in the case $f \in C^{0}(G)$ and $g \in H^{\Gamma}(G) \cap C^{k-1}(\bar{G})$ ) the function $u=v+g \in H^{\Gamma}(G)$ has more regularity, $u \in X$, and if $G$ has Property $\mathrm{P}$, the function $u$ is also a classical solution of the differential equation (3.1a) in $G$ and fulfils the boundary condition (3.1b) in the usual sense (cf. Theorem 4).

\section{On the regularity of the solutions of the generalized}

4.1. Let $G$ be a bounded open set with Property P. Furthermore, let $f \in L^{2}(G)$ and an "admissible" boundary data $g \in H^{\Gamma}(G)$ be given. We call the boundary data $g \in H^{\Gamma}(G)$ admissible if the linear functional $l_{f, g}$ is also bounded on $L^{2}(G)$, and therefore by the Fréchet-Riesz theorem there exists an element $h \in L^{2}(G)$ such that the relation

$$
l_{f, g}(\varphi)=(h, \varphi)_{0}
$$

holds for all $\varphi \in C_{0}^{\infty}(G)$.

First we prove, in the case where the function $h \in L^{2}(G)$ has the form

$$
h=h_{1} \ldots h_{k}
$$

with $h_{j} \in L^{2}\left(G_{j}\right)(1 \leqq j \leqq k)$, a regularity result which is similar to that of [3] (pp. 46-68). At the second stage we will drop assumption (4.2) and take an arbitrary $h \in L^{2}(G)$.

We denote by $H_{0}^{1}\left(G_{j}\right)(1 \leqq j \leqq k)$ the usual Sobolev space of functions with generalized homogeneous boundary values and by $\|\cdot\|_{1, G_{j}}$ the norm in $H_{0}^{1}\left(G_{j}\right)$. For $1 \leqq j \leqq k$ let $B_{j}$ be the sesquilinear form

$$
B_{j}\left(\varphi_{j}, \psi_{j}\right):=\int_{G_{j}} \sum_{i=1}^{m_{j}} \overline{D_{l_{j}+i} \varphi_{j}\left(x^{(j)}\right)} D_{l_{j}+i} \psi_{j}\left(x^{(j)}\right) d x^{(j)}
$$

defined for $\varphi_{j}, \psi_{j} \in C_{0}^{\infty}\left(G_{j}\right) \quad$ with $\quad x^{(j)}:=\left(x_{l_{j}+1}, \ldots, x_{l_{j}+m_{j}}\right)$ and $d x^{(j)}:=$ $d x_{l_{j}+1} \ldots d x_{l_{j}+m_{j}}$. This form gives a norm $\|\mid\| \|_{1_{1, G_{j}}}$,

$$
\left\|\mid \varphi_{j}\right\|_{1, G_{j}}^{2}=B_{j}\left(\varphi_{j}, \varphi_{j}\right)
$$

equivalent to $\|\cdot\|_{1, G_{j}}$.

By the theory of elliptic differential operators there exists for each $j(1 \leqq j \leqq k)$ a unique $v_{j} \in H_{0}^{1}\left(G_{j}\right)$ such that

$$
\left(h_{j}, \varphi_{j}\right)_{0, G_{j}}=B_{j}\left(v_{j}, \varphi_{j}\right)
$$

holds for all $\varphi_{j} \in C_{0}^{\infty}\left(G_{j}\right)$. 
Theorem 8. Let $G$ be a bounded open set with Property P. Let $f \in L^{2}(G)$ and an admissible boundary data $g \in H^{\Gamma}(G)$ be given. Furthermore assume that the element $h \in L^{2}(G)$ representing the functional $l_{f, g}$ of Problem 2 has the form (4.2). Then the unique solution of Problem 2 is the product of the unique solutions of the equations (4.5).

Proof. A. First we prove by induction that the product $v=v_{1} \ldots v_{k}$ of the solutions $v_{j}$ of the equations (4.5) belongs to $H_{0}^{T}(G)$. For $k=1$ this is trivial because then the equality $H_{0}^{1}(G)=H_{0}^{T}(G)$ holds. For $l \in N, 1 \leqq l \leqq k$, we define

$$
\Gamma_{l}=\left\{\alpha \mid \alpha \in N_{0}^{n}, \alpha=\sum_{j=1}^{l} \varepsilon_{t_{j}}\right\}
$$

(cf. (1.3)). Note that with this definition we have $\Gamma_{k}=\Gamma$.

Now let $G^{\wedge}:=G_{1} \times \ldots \times G_{l-1}, l \leqq k$, and assume $\tilde{v}=v_{1} \ldots v_{l-1} \in H_{0}^{l-1}\left(G^{\wedge}\right)$ with $v_{j} \in H_{0}^{1}\left(G_{j}\right), 1 \leqq j \leqq l-1$. Thus, there exists a sequence $\left\{\tilde{\varphi}_{m}\right\}_{m \in N}, \tilde{\varphi}_{m} \in C_{0}^{\infty}\left(G^{\wedge}\right)$ such that

$$
\left\|\tilde{v}-\tilde{\varphi}_{m}\right\|_{\Gamma_{l-1}, G^{\wedge}} \rightarrow 0
$$

holds for $m \rightarrow \infty$. On the other hand, since $C_{0}^{\infty}\left(G_{l}\right)$ is dense in $H_{0}^{1}\left(G_{l}\right)$, we can find a sequence $\left\{\varphi_{l, m}\right\}_{m \in N}, \varphi_{l, m} \in C_{0}^{\infty}\left(G_{l}\right)$, such that

$$
\left\|\left|v_{l}-\varphi_{l, m}\right|\right\|_{1, G_{l}} \rightarrow 0
$$

holds for $m \rightarrow \infty$. Now, if we put $G^{\prime}=G^{\wedge} \times G_{l}(l \leqq k)$,

$$
\begin{aligned}
& \left\|\left|\tilde{v}-\tilde{\varphi}_{m}\right|\right\|_{\Gamma_{l-1}, G^{\wedge}}\left\||| v_{l}-\varphi_{l, m}\right\|_{\left.\right|_{1, G_{l}}}=\|\|\left(\tilde{v}-\tilde{\varphi}_{m}\right)\left(v_{l}-\varphi_{l, m}\right) \| \Gamma_{l}, G^{\prime} \\
& \geqq\left.\left\|\left|\tilde{v} v_{l}-\tilde{\varphi}_{m} \varphi_{l, m}\right|\right\|\right|_{\Gamma_{l}, G^{\prime}}-\left\||| \tilde{\varphi}_{m}\left|\left\|_{\Gamma_{l-1}, G^{\wedge}}\right\|\left\|v_{l}-\varphi_{l, m}\right\|\left\|_{1, G_{l}}-\right\|\right|\left|\varphi_{l, m}\left\|_{1, G_{l}}\right\|\right| \tilde{v}-\tilde{\varphi}_{m} \mid\right\|_{\Gamma_{l-1}, G^{\wedge}} .
\end{aligned}
$$

Since $\left\|\tilde{\varphi}_{m}\right\| \|_{\Gamma_{l-1}, G^{\wedge}}$ and $\left\|\mid \varphi_{l, m}\right\| \|_{1, G_{l}}$ are bounded it follows by (4.6) and (4.7) that

for $m \rightarrow \infty$.

$$
\left\|\tilde{v} v_{l}-\tilde{\varphi}_{m} \varphi_{l, m}\right\|_{\Gamma_{l}, G^{\prime}} \rightarrow 0
$$

B. Next we prove that $v=v_{1} \ldots v_{k}$ solves the equation

$$
B(v, \varphi)=\left(h_{1} \ldots h_{k}, \varphi\right)_{0, G}
$$

for all $\varphi \in C_{0}^{\infty}(G)$. For the functions $\varphi_{j} \in C_{0}^{\infty}\left(G_{j}\right) \quad(1 \leqq j \leqq k)$ we have by (4.3) and (4.5)

$$
\begin{gathered}
B\left(v_{1} \ldots v_{k}, \varphi_{1} \ldots \varphi_{k}\right)=B_{1}\left(v_{1}, \varphi_{1}\right) \ldots B_{k}\left(v_{k}, \varphi_{k}\right) \\
=\left(h_{1}, \varphi_{1}\right)_{0, G_{1}} \ldots\left(h_{k}, \varphi_{k}\right)_{0, G_{k}}=\left(h_{1} \ldots h_{k}, \varphi_{1} \ldots \varphi_{k}\right)_{0, G} .
\end{gathered}
$$


By [9] (Corollary 1 and 2 of Theorem 39.2, p. 409) the equation (4.8) can be extended continuously to all elements $\varphi \in C_{0}^{\infty}(G)$ instead of $\varphi_{1} \ldots \varphi_{k}$. Thus $v$ is the unique solution of Problem 2.

C. Assuming the boundaries $\partial G_{j}(1 \leqq j \leqq k)$ to be smooth enough we can apply the regularity theory for solutions of elliptic equations (cf. [3], pp. 46-68) to the functions $v_{j} \in H_{0}^{1}\left(G_{j}\right)(1 \leqq j \leqq k)$ solving (4.5). Hence for smooth boundaries $\partial G_{j}$ and $h_{j} \in L^{2}\left(G_{j}\right)$ we find $v_{j} \in H^{2}\left(G_{j}\right) \cap H_{0}^{1}\left(G_{j}\right)(1 \leqq j \leqq k)$ and therefore

$$
v_{1} \ldots v_{k}=v \in H^{2 \Gamma}(G) \cap H_{0}^{\Gamma}(\boldsymbol{G}) .
$$

If in addition $h_{j} \in C^{\infty}\left(\bar{G}_{j}\right)(1 \leqq j \leqq k)$, we have (again under the assumption of smooth boundaries) $v_{j} \in H_{0}^{1}\left(G_{j}\right) \cap C^{\infty}\left(\bar{G}_{j}\right)$ and therefore

$$
v_{1} \ldots v_{k}=v \in H_{0}^{\Gamma}(G) \cap C^{\infty}(\bar{G})
$$

(see [3], p. 68).

4.2. We will now examine the regularity of the solution of Problem 2 for arbitrary admissible data.

Theorem 9. Let $G$ be a bounded open set with Property P. Furthermore let $f \in L^{2}(G)$ and an admissible $g \in H^{\Gamma}(G)$ be given. Then the unique solution $v$ of Problem 2 belongs to $H^{(k)}(G)$.

Proof. Since $g$ is admissible there exists an element $h \in L^{2}(G)$ such that the functional $l_{f, g}$ of Problem 2 has the representation $l_{f, g}(\varphi)=(h, \varphi)_{0}$. By [9] (Corollary 1 of Theorem 39.2, p. 409) there exists for $h$ and for each $\varepsilon \in \boldsymbol{R}, \varepsilon>0$ a function $h_{\varepsilon} \in C_{0}^{\infty}(G)$ such that

$$
\left\|h-h_{\varepsilon}\right\|_{0, G}<\varepsilon
$$

holds, where $h_{\varepsilon}$ has the form

$$
h_{\varepsilon}:=\sum_{\left(i_{1}, \ldots, i_{k}\right) \in \Omega} h_{i_{1}, \varepsilon} \ldots h_{i_{k}, \varepsilon}
$$

with a suitable finite subset $\Omega$ of $N^{k}$ and $h_{i_{j}, \varepsilon} \in C_{0}^{\infty}\left(G_{j}\right)(1 \leqq j \leqq k)$.

For the functions $h_{i_{j}, \varepsilon}(1 \leqq j \leqq k)$ we consider the unique solutions $v_{i_{j}, \varepsilon} \in H_{0}^{1}\left(G_{j}\right)$ of the generalized strongly elliptic Dirichlet problems

$$
\left(h_{i_{j}, \varepsilon}, \varphi\right)_{0, G_{j}}=B_{j}\left(v_{i_{j}, \varepsilon}, \varphi_{j}\right) \text { for all } \varphi_{j} \in C_{0}^{\infty}\left(G_{j}\right) .
$$

From the elliptic theory we get $v_{i_{j}, \varepsilon} \in C^{\infty}\left(\bar{G}_{j}\right)$ (cf. [3], p. 68). The function

$$
v_{\varepsilon}:=\sum_{\left(i_{1}, \ldots, i_{k}\right) \in \Omega} v_{i_{1}, \varepsilon} \ldots v_{i_{k}, \varepsilon} \in H_{0}^{\Gamma}(G) \cap C^{\infty}(\bar{G})=X=D(L)
$$


is the unique solution of the equation

$$
\left(h_{\varepsilon}, \varphi\right)_{0, G}=B\left(v_{\varepsilon}, \varphi\right) \text { for all } \varphi \in C_{0}^{\infty}(G) .
$$

We denote the unique solution of Problem 2 again by $v \in H_{0}^{\Gamma}(G)$. We have

$$
B\left(v-v_{\varepsilon}, \varphi\right)=\left(h-h_{\varepsilon}, \varphi\right)_{0, G} \text { for all } \varphi \in C_{0}^{\infty}(G) .
$$

The continuity of the sesquilinear form $B$ in $H_{0}^{T}(G)$ implies (with $v-v_{\varepsilon}$ instead of $\varphi$ )

$$
\left\|v-v_{\varepsilon}\right\|_{\Gamma}^{2} \leqq\left\|h-h_{\varepsilon}\right\|_{0}\left\|v-v_{\varepsilon}\right\|_{0} \leqq c\left\|h-h_{\varepsilon}\right\|_{0}\left\|v-v_{\varepsilon}\right\|_{\Gamma}
$$

with a positive constant $c$. We apply (4.11) and get

$$
\left\|v-v_{\varepsilon}\right\|_{\Gamma} \leqq c \varepsilon .
$$

Thus, because of the equivalence of the norms $\|\cdot\|_{\Gamma}$ and $\|\cdot\| \|_{\Gamma}$ on $H_{0}^{\Gamma}(G)$ we have

for $\varepsilon \rightarrow 0$, and by (4.11)

$$
\left\|v-v_{\varepsilon}\right\|_{0} \rightarrow 0
$$

$$
\left\|h-L v_{\varepsilon}\right\|_{0}=\left\|h-h_{\varepsilon}\right\|_{0} \rightarrow 0 .
$$

Since $L^{\sim}$ is the closure of $L$ we get finally

$$
v \in D\left(L^{\sim}\right)=H^{(k)}(G) .
$$

\section{References}

[1] DoLPH, C. L.: The mathematician grapples with linear problems associated with the radiation condition. - Proceedings of the Symposium on electromagnetic wave theory, The University of Michigan 1955. IRE Trans. Antennas and Propagation 4, 1956, 302-311.

[2] Dynkin, E. B.: Harmonic functions associated with several Markov processes. - Adv. in Appl. Math. 2, 1981, 260-283.

[3] Friedman, A.: Partial differential equations. - Holt, Rinehart and Winston, Inc., New YorkChicago-etc., 1969. Reprint: Robert E. Krieger Publishing Company, Huntington (New York), 1976.

[4] Hess, P.: Über das verallgemeinerte Dirichletproblem für lineare partielle Differentialgleichungen. - Ann. Acad. Sci. Fenn. Ser. A. I. Math. 434, 1969, 1-28.

[5] Hildebrandt, S.: Lineare elliptische Differentialgleichungen. - [Vorlesung, Mainz 1966, als Manuskript vervielfältigt.] Johannes Gutenberg -Universität, Mathematisches Institut, Mainz, 1966.

[6] Hörmander, L.: Linear partial differential operators. - Die Grundlehren der mathematischen Wissenschaften 116. Springer-Verlag, Berlin-Göttingen-Heidelberg, 1963.

[7] JАСOB, N.: On generalized Dirichlet problems. - Math. Scand. [To appear.] 
[8] Nevanlinna, R.: Erweiterung der Theorie des Hilbertschen Raumes. - Medd. Lunds Univ. Mat. Sem. Supplementband tillägnat Marcel Riesz, Tome supplémentaire dédié à Marcel Riesz, 1952, 160-168.

[9] Trèves, F.: Topological vector spaces, distributions and kernels. - Pure and Applied Mathematics 25. Academic Press, New York-London, 1967.

[10] Weidmann, J.: Lineare Operatoren in Hilberträumen. - Mathematische Leitfäden. B. G. Teubner, Stuttgart, 1976.

Freie Universität Berlin

Fachbereich Mathematik

Arnimallee 2-6

D 1000 Berlin 33

Bundesrepublik Deutschland
New addres of N. J.:

Hochschule der Bundeswehr Müncheu Fachbereich Informatik

Institut für Mathematik

Werner-Heisenberg-Weg 39

D 8014 Neubiberg

Bundesrepublik Deutschland

Received 12 August 1983 